\title{
The incidence and clinical relevance of anti-HLA and/or MICA antibodies in patients with long-term survival renal allo-grafts
}

\author{
Dongmei Wang, Xiaoyan Shan, Lijun Wang, Wei Li, Na Liu, Dongmei Li, Yuanyuan Jing, Yanjun Jia* \\ Department of HLA, Beijing Red Cross Blood Center, Beijing 100088, China
}

\begin{abstract}
The purpose of this study was to analyze the incidence and clinical relevance of anti-HLA and/or MICA antibodies in renal allo-graft transplant recipients with long-term renal survival ( $>5$ years). This retrospective study collected post-transplant serum samples from a total of 110 patients which were used to detect the incidence of anti-HLA and/or MICA antibodies as well as anti-HLA donor specific antibodies. Among these 110 patients, 72 patients had antibodies against HLA and/or MICA at the time of test, 61 had anti-HLA antibodies, 31 had anti-MICA antibodies, and 38 were antibody negative. There was no difference in the number of patients developing antibodies against non-donor specific antibodies, donor specific antibodies, Class I donor specific antibodies, Class II donor specific antibodies or MICA antibodies between normal function group (serum creatinine level $<2.0 \mathrm{mg} / \mathrm{dL}$ ) and dysfunction group (serum creatinine level $>2.0 \mathrm{mg} / \mathrm{dL}$ ). For the serum creatinine level, estimated glomerular filtration rate and blood urea nitrogen level, patients with different antibodies were not statistically different to antibodynegative patients. Cox regression analysis showed that the type of transplantation and HLA mismatch number were significant negative risk factors for the development of anti-HLA $(P<0.05)$. Our results suggested that the antiHLA antibody status has little impact on the renal graft function in the long-term survival allo-graft renal recipients.
\end{abstract}

Keywords: HLA, DSA, MICA, renal transplantation

\section{INTRODUCTION}

In the past two decades, long-term renal graft survival has been greatly improved by the use of more effective immunosuppressive drugs and therapeutic regimens. However, immunological and non-immunological factors still exist which can lead to chronic renal allograft dysfunction and loss ${ }^{[1]}$. Several reports have suggested that the humoral response to alloantigens is one of the reasons for the development of chronic allograft nephropathy and rejection ${ }^{[2-4]}$.

The role of antibodies against human leukocyte an-

*Correspondence to: Yanjun Jia, Department of HLA, Beijing Red Cross Blood Center, No. 37 Beisanhuan Zhong Road, Beijing 100088, China. TEL: +86-10-82807279, E-mail: jiayj321@ hotmail.com.

Confiict of interests: The authors have declared no conflict of interests. tigens (anti-HLA Abs), especially anti-HLA donorspecific antibodies (HLA DSA) in the failure of kidney allografts has been understood more clearly over the past decade ${ }^{[5-7]}$. The presence of anti-HLA Abs is a major factor causing acute-mediated rejection and also may lead to chronic antibody-mediated allograft rejection ${ }^{[8]}$.

In addition to anti-HLA Abs, antibodies against MHC Class I-related Chain A antigen (anti-MICA Abs) also play an important role in the renal allograft rejection, which develops more frequently in patients with graft failure than in those with functioning grafts ${ }^{[9]}$. Accumulated data shows that specific anti-MICA Abs are related to allograft dysfunction and rejection, even if anti-HLA Abs are absent ${ }^{[10]}$. MCIA is a member of the non-classical HLA Class- I family with a large degree of polymorphism expressed on the surfaces of dendritic cells, monocytes, activated $\mathrm{T}$ cells, keratino- 
cytes, fibroblasts, endothelial and epithelial cells ${ }^{[11-13]}$.

The introduction of the Luminex solid-phase technique allows us to detect with more specificity and higher sensitivity HLA Abs and MICA Abs which play detrimental roles in immunologic processes causing renal allograft rejection ${ }^{[14,15]}$. The purpose of this study was to investigate the prevalence of antiHLA and anti-MICA Abs in renal transplantation patients with long-term survival of over 5 years by using Luminex solid-phase assay. The correlation of the presence of antibodies against HLA, DSA and MICA with renal function was also investigated.

\section{MATERIALS AND METHODS}

\section{Patients} long-term survival of over 5 years who experienced renal transplantation from 1993 to 2009 at different hospitals and transplant centers. The study was approved by the institutional review boards of Beijing Red Cross Blood Center and all patients signed the written informed consent. The mean age of 110 patients at the time of transplantation as well as their sex, months after transplantation, donor type (deceased or living), HLA mismatch number and therapeutic regimen [Tacrolimus (FK506) or Cyclosporine (CsA) plus mycophenolatemofetil (MMF) and prednisone] are shown in Table 1. The HLA typing of recipients and donors for HLA-A, -B, -DR and -DQ was available for all cases so the DSA for HLA could be determined but not for MICA.

Table 1 General characteristics of patients with and without anti-HLA and/or MICA antibodies

\begin{tabular}{|c|c|c|c|c|c|}
\hline Characteristics & $\begin{array}{c}\text { HLA and/or MICA } \\
\text { Abs }(n=72)\end{array}$ & $\begin{array}{l}\text { HLA Abs } \\
(n=61)\end{array}$ & $\begin{array}{c}\text { MICA Abs } \\
(n=31)\end{array}$ & $\begin{array}{c}\text { Antibody negative } \\
(n=38)\end{array}$ & $P^{*}$ \\
\hline Age at time of transplant (year, mean \pm SD) & $39.82 \pm 11.13$ & $39.92 \pm 11.24$ & $39.06 \pm 9.19$ & $37.24 \pm 12.34$ & NS \\
\hline Gender, male $(n)$ & 33 & 27 & 14 & 21 & NS \\
\hline Scr level(mg/dL, mean $\pm \mathrm{SD})$ & $2.74 \pm 2.18$ & $2.84 \pm 2.27$ & $2.40 \pm 1.43$ & $2.33 \pm 1.34$ & NS \\
\hline $\mathrm{eGFR}^{\mathrm{a}}($ mean $\pm \mathrm{SD})$ & $39.07 \pm 27.98$ & $37.51 \pm 27.78$ & $40.43 \pm 26.19$ & $45.19 \pm 32.62$ & NS \\
\hline Baseline immunosuppression (count) & & & & & NS \\
\hline CsA/MMF/prednisone & 61 & 51 & 29 & 21 & \\
\hline FK506/MMF/prednisone & 11 & 10 & 2 & 17 & \\
\hline BUN level(mmol/L, mean $\pm \mathrm{SD})$ & $12.65 \pm 6.70$ & $12.91 \pm 6.93$ & $11.97 \pm 5.42$ & $13.57 \pm 9.32$ & NS \\
\hline HLA mismatch number $(n$, mean \pm SD) & $4.58 \pm 1.71$ & $4.70 \pm 1.76$ & $4.12 \pm 1.36$ & $2.97 \pm 1.62$ & $<0.01$ \\
\hline
\end{tabular}

\section{Detection of anti-HLA and MICA Abs}

Sera samples were collected when these patients came to hospital for follow-up review from May 2014 to November 2014. Each serum sample was screened firstly for HLA Class I, Class II and MICA antibodies with LABScreen Mixed beads (One Lambda, Inc., Canoga Park, CA). If showing positive results, the sera of patients were then tested by LABScreen Single Antigen beads (One Lambda, Inc., Canoga Park, CA). All tests were performed according to the manufacturer's recommendation. Briefly, $20 \mu \mathrm{L}$ test serum were added to 96 -well micro-plate with $5 \mu \mathrm{L}$ beads in each well, then incubated for $30 \mathrm{~min}$ at room temperature in the dark place, and washed 3 times with wash buffer; $100 \mu \mathrm{L}$ goat anti-human IgG secondary antibody labeled with R-phycoerythrin (PE) were added and incubated for $30 \mathrm{~min}$ in the dark at room temperature, and then washed 3 times again with wash buffer and read on the LABScan 100 flow cytometer (One Lambda, Inc.). A negative control serum (LSNC; One Lambda, Inc.) was also included in each assay, which was used to normalize the mean fluorescence intensity (MFI) of test samples and calculate the normalized background ratio (NBG ratio). NBG ratios $>2.2$ was considered as positive results. The NBG ratio was calculated by the following formula: NBG ratio $=(\mathrm{S \# N}$ -SNC bead)/(BG\#N-BGNC bead). In which, S\#N represents sample-specific fluorescent value for bead \#N; SNC bead represents sample-specific fluorescent value for Negative Control bead; BG\#N represents background $\mathrm{NC}$ serum fluorescent value for bead \#N and BGNC bead represents background NC serum fluorescent value for Negative Control bead.

\section{Measurement of renal function}

The serum creatinine level (Scr, mg/dL) and blood urea nitrogen level (BUN, mmol/L) in the blood samples of patients were tested by Hitachi $7060 \mathrm{Au}-$ tomatic Biochemical Analyzer. The estimated glomerular filtration rate (eGFR) was calculated with the equation published previously by Schwartz et al. ${ }^{[16]}$. These three measurements were used to assess renal graft function. 
The incidence and clinical relevance of anti-HLA and/or MICA antibodies in patients with long-term 47 survival renal allo-grafts, 2018, 2(1)

\section{Statistical analysis}

The continuous variables were expressed as means standard deviations and discrete variables were presented as numbers or percentages. The chi-square test was used to compare the categorical data and the unpaired $t$ test or one-way ANOVA analysis was applied for the continuous data. Two-sided $P$ values less than 0.05 were considered significant. Risk factors for antiHLA DSA Abs production were assessed using Cox proportional hazard regression analyses in which the following potential confounders were considered: the year of transplantation; the age of recipients; the type of transplantation (living or deceased); the patient's sex; number of HLA-A, -B, -DR, -DQ mismatches; type of immunosuppression (FK506/MMF/Prednisone or CsA/MMF/prednisone), and eGFR at the time of testing. Factors found to be significant on univariate analysis $(P$ value $<0.1)$ were introduced into a backward stepwise Cox regression model (multivariate analyses). Final multivariate analysis factors are reported as a hazard ratio with a $95 \%$ confidence interval. The software packages SPSS (version 14.0) was used to process data and perform statistical analyses.

\section{RESULTS}

\section{General characteristics of patients with long- term survival}

The general characteristics of all patients with survival of over 5 years are shown in Table 1 according to their different HLA and/or MICA Abs status. The enrolled 110 renal transplants were divided into four groups including anti-HLA and MICA Abs positive group, anti-HLA Abs positive group, antiMICA Abs positive group and antibody negative group. As shown in Table 1, 72 patients had antiHLA Abs and/or MICA Abs at the time of test, 61 had anti-HLA Abs, 31 had anti-MICA Abs, and 38 were antibody negative. There was no significant difference among these groups in terms of age at time of transplant, gender proportions and baseline immunosuppression. Although the mean value of Scr level, eGFR or BUN level was higher in the antibody positive group, there was also no statistically significance among the 4 groups as tested by one-way ANOVA method. The patients with anti-HLA or MICA Abs had a significantly longer post-transplant survival time than those without antibodies. Moreover, the HLA mismatch number would significantly affect the development of antiHLA or MICA Abs because there was a statistically significant difference when comparing this variable among four groups.

\section{The incidence and specificity of anti-HLA and MICA Abs in renal transplant patients}

A Luminex Single Bead kit was used to determine the specificity of anti-HLA Abs in the serum showing anti-HLA Abs positive results on mixed beads. All patients were divided into two groups according to their serum creatinine level, i.e. the normal group (with Scr level lower than $2.0 \mathrm{mg} / \mathrm{dL}$ ) and dysfunction group (with Scr level above $2.0 \mathrm{mg} / \mathrm{dL}$ ).

The incidence and specificity of anti-HLA and MICA Abs in patients with or without normal renal graft function is listed in Table 2. 22 (51.16\%) of the patients with normal renal function developed antiHLA Abs, including $4(9.30 \%)$ non-donor specific anti-HLA antibodies (NDSA) and $18(41.86 \%)$ DSA; $5(11.63 \%)$ developed anti-MICA Abs. In contrast, 39 $(58.21 \%)$ of patients from the dysfunction group produced anti-HLA Abs including 13(19.40\%) NDSA and $26(38.80 \%)$ DSA; 10 (14.93\%) produced antiMICA Abs. Among the HLA DSA in the graft functioning group, $10(23.26 \%)$ were HLA Class I and 9 (20.93\%) were HLA Class II. The numbers of patients with HLA Class I and Class II DSA in the graft dysfunction group were 8 (11.94\%) and 21 (31.34\%) respectively. Between two groups, there was no difference in the number of patients developing NDSA, DSA, Class I DSA, Class II DSA or MICA Abs. The highest mean signal intensity of antibodies as measured by the MFI was higher in the dysfunction group, but there was no statistically significant dif-

Table 2 The incidence and specificity of anti-HLA and MICA Abs in the patient with or without normal renal graft function

\begin{tabular}{|c|c|c|c|c|c|c|}
\hline \multirow{2}{*}{ Antibody status } & \multicolumn{2}{|c|}{ Normal group $(n=43)$} & \multicolumn{2}{|c|}{ Dysfunction group $(n=67)$} & \multirow{2}{*}{$P$ value ${ }^{*}$} & \multirow{2}{*}{ Total $(n=110)$} \\
\hline & Cases $(\%)$ & MFI (mean $\pm S D)$ & Cases $(\%)$ & MFI (mean $\pm S D)$ & & \\
\hline HLA & $22(51.16)$ & & $39(58.21)$ & & & \\
\hline NDSA & $4(9.30)$ & $3,110 \pm 5,334$ & $13(19.40)$ & $3,116 \pm 4,394$ & 0.9982 & 17 \\
\hline DSA & $18(41.86)$ & $1,460 \pm 2,441$ & $26(38.80)$ & $5,331 \pm 5,586$ & 0.0086 & 44 \\
\hline Class I & $10(23.26)$ & $997 \pm 362$ & $8(11.94)$ & $2,167 \pm 3,207$ & 0.2657 & 18 \\
\hline Class II & $9(20.93)$ & $2,813 \pm 4,643$ & $21(31.34)$ & $5,844 \pm 5,886$ & 0.1820 & 30 \\
\hline
\end{tabular}

${ }^{*} P$ value indicates the difference of MFI between two groups. 
48 The incidence and clinical relevance of anti-HLA and/or MICA antibodies in patients with long-term survival renal allo-grafts, 2018, 2(1)

ference between the two groups in terms of NDSA, Class I DSA, Class II DSA and MICA. The difference in average MFI was statistically significant only in the case of HLA DSA. The MFI of HLA DSA in patients with dysfunctional renal grafts was much higher than those in patients with normal renal function $(1,460 \pm 2,441$ vs. $5,331 \pm 5,586, P=0.0086)$. Although there was no statistically significant difference between normal and dysfunction groups in terms of anti-MICA Abs (owing to the small sample number and large standard deviation), a large difference was found between their mean MFI $(752 \pm 541$ vs.5,575 $\pm 7,191, P=0.1654)$.

Table 3 displays the characteristics of all DSA(+) patients including those with HLA Class I and II Abs status, HLA ABDRDQ mismatch with donor and specific type of HLA DSA against each type of Class I and Class II molecules with the correspond-

Table 3 Characterization of donor specific antibody (DSA) positive patients

\begin{tabular}{|c|c|c|c|c|}
\hline $\begin{array}{l}\text { DSA (+) } \\
\text { patient ID }\end{array}$ & $\begin{array}{c}\text { Class I and II Abs } \\
\text { status } \\
\end{array}$ & $\begin{array}{c}\text { HLA MM with donor } \\
\text { (A/B/DR/DQ) }\end{array}$ & DSA Class I (MFI) & DSA Class II (MFI) \\
\hline 1 & $-1+$ & 2/1/1/- & - & DR4 $(3,144)$ \\
\hline 2 & $-1+$ & $0 / 2 / 2 / 1$ & - & DR9 (556), DQ9 (521) \\
\hline 3 & $+1-$ & $1 / 2 / 1 / 1$ & B60 (395) & - \\
\hline 4 & $-1+$ & $2 / 2 / 2 / 2$ & - & DR7 $(1,636)$, DQ4 $(1,219)$ \\
\hline 5 & $-1+$ & $1 / 2 / 2 / 2$ & - & DR4 (10,795), DR15 $(9,396)$, DQ6 $(6,413)$, DQ8 $(10,436)$ \\
\hline 6 & $+/-$ & $1 / 1 / 0 / 0$ & A2 (1,625), A75 (964) & - \\
\hline 7 & $-1+$ & $2 / 2 / 1 / 1$ & - & DR17 (1394), DQ2 (489) \\
\hline 8 & $-1+$ & $2 / 2 / 2 / 1$ & - & DR9 (534), DR10 (4,565), DQ9 $(2,306)$ \\
\hline 9 & $+1-$ & $1 / 1 / 0 / 1$ & B75 $(2,884)$ & - \\
\hline 10 & $-1+$ & $2 / 2 / 1 / 1$ & - & DR17 $(1,592)$ \\
\hline 11 & $+/+$ & $2 / 2 / 2 /-$ & - & DR4 (250), DR12 (331) \\
\hline 12 & $+/-$ & 1/1/1/- & A11 (1,016), B48 (298) & - \\
\hline 13 & $-1+$ & $1 / 1 / 2 / 1$ & - & DR12 $(9,086)$, DR15 $(3,470)$ \\
\hline 14 & $+/-$ & $2 / 1 / 0 /-$ & A2 $(1,593)$ & - \\
\hline 15 & $-1+$ & $0 / 2 / 1 / 1$ & - & DR8 $(6,998)$, DQ4 $(8,726)$ \\
\hline 16 & $+/-$ & $1 / 2 / 1 / 1$ & B7 (613) & - \\
\hline 17 & $+1-$ & 1/1/1/- & A11 (1,455), B48 (473) & - \\
\hline 18 & $+/+$ & $1 / 2 / 2 / 1$ & A30 (577) & DR7 (418),DR12 (768) \\
\hline 19 & $-1+$ & $1 / 1 / 1 /-$ & - & DR15 (409) \\
\hline 20 & $-1+$ & $2 / 2 / 2 / 1$ & - & DR9 $(1,482)$, DR10 $(8,839)$, DQ9 $(5,410)$ \\
\hline 21 & $+/-$ & $1 / 1 / 1 / 2$ & A33 (764), B52 (355) & - \\
\hline 22 & $+/ 1$ & $2 / 2 / 1 / 1$ & A2 (708), B54 (421) & - \\
\hline 23 & $-1+$ & $2 / 0 / 1 / 2$ & - & DR12 (7,519), DQ7 (8,321), DQ8 $(3,399)$ \\
\hline 24 & $-1+$ & 1/1/1/- & - & DR15 (336) \\
\hline 25 & $-1+$ & $2 / 2 / 2 / 1$ & - & DR9 (1,314), DR10 (8,014), DQ9 $(4,782)$ \\
\hline 26 & $+/-$ & $1 / 1 / 1 / 2$ & A33 (403) & - \\
\hline 27 & $-1+$ & $0 / 2 / 1 / 1$ & - & DR8 $(8,038)$, DQ4 $(9,229)$ \\
\hline 28 & $+/+$ & $1 / 2 / 1 / 1$ & B7 (595) & DR4 $(1,138)$ \\
\hline 29 & $-1+$ & $1 / 2 / 2 / 2$ & - & DR4 (9,879), DR15 (9,464), DQ6 (6,805), DQ8 $(7,382)$ \\
\hline 30 & $+1-$ & $2 / 1 / 0 /-$ & A2 $(1,037)$, B48 (452) & - \\
\hline 31 & $-1+$ & $1 / 1 / 2 / 1$ & - & DR12 (8,408), DR15 $(2,801)$ \\
\hline 32 & $+1-$ & $1 / 1 / 1 /-$ & A11 (748) & - \\
\hline 33 & $-1+$ & $0 / 1 / 2 / 1$ & - & DR7 (521), DR17 (834), DQ4 (358) \\
\hline 34 & $+/+$ & $2 / 2 / 1 / 1$ & A2 (804) & DR13 (205) \\
\hline 35 & $-1+$ & $0 / 1 / 1 /-$ & - & DR15 (883) \\
\hline 36 & $+/+$ & $1 / 1 / 1 /-$ & Al1 $(9,836)$, B48 $(1,953)$ & DR12 (265) \\
\hline 37 & $-1+$ & $1 / 1 / 1 /-$ & - & DR15 (366) \\
\hline 38 & $+/-$ & $2 / 2 / 1 / 1$ & A2 (505), B54 (792) & - \\
\hline 39 & $-1+$ & $1 / 2 / 2 / 2$ & - & DR4 (9,779), DR15 $(8,375)$, DQ6 $(10,686)$, DQ8 $(11,032)$ \\
\hline 40 & $+/-$ & 2/1/0/- & A2 $(1,203)$, B48 (501) & - \\
\hline 41 & $-1+$ & $1 / 1 / 1 /-$ & - & DR15 (286) \\
\hline 42 & $-1+$ & $0 / 2 / 1 / 1$ & - & DR8 $(6,620)$, DQ4 $(8,796)$ \\
\hline 43 & $+/+$ & $1 / 2 / 1 / 1$ & $\begin{array}{c}\text { A24 }(16,527), \mathrm{B} 46(20,077), \mathrm{B} 35 \\
(24,296)\end{array}$ & DR4 $(24,514), \mathrm{DQ9}(23,185)$ \\
\hline 44 & $-1+$ & 1/1/1/- & - & DR15 (874) \\
\hline
\end{tabular}


ent MFI value. There were 44 patients who developed HLA DSA after transplantation. In these 44 DSApositive patients, 18 had HLA Class I DSA and 30 patients had HLA Class II DSA, whereas 6 displayed both HLA class I and II DSA.

\section{The correlation of antibody status with renal allo-graft function}

The Scr level, eGFR calculated according to Scr, and BUN level are three important indicators for re- nal graft function. Table 4 displays their values in patients with different antibody statuses. For the Scr, eGFR and BUN level, patients with anti-HLA and/ or MCIA Abs, anti-HLA Abs, anti-HLA DSA, antiClass I DSA, anti-Class II DSA or MICA Abs were not statistically different to antibody-negative patients. These results implied that the levels of antiHLA and/or MICA antibodies had no direct association with renal graft function in long-term survival renal recipients.

Table 4 Comparison of eGFR between anti-HLA and/or MICA antibody- positive and negative patients

\begin{tabular}{lcccccc}
\hline HLA and/or MICA antibody status & Scr $(\mathrm{mg} / \mathrm{dL})$ & $P^{*}$ & eGFR $\left(\mathrm{mL} / \mathrm{min} / 1.73 \mathrm{~m}^{2}\right)$ & $P^{*}$ & BUN $(\mathrm{mmol} / \mathrm{L})$ & $P$ \\
\hline Antibody negative $(n=38)$ & $2.33 \pm 1.34$ & - & $45.18 \pm 32.62$ & - & $13.57 \pm 9.32$ & - \\
HLA and/or MICA Abs positive $(n=72)$ & $2.74 \pm 2.18$ & 0.2927 & $39.07 \pm 27.98$ & 0.3064 & $12.83 \pm 6.70$ & 0.6326 \\
HLA Abs positive $(n=61)$ & $2.84 \pm 2.27$ & 0.2128 & $37.51 \pm 27.78$ & 0.2147 & $13.13 \pm 6.93$ & 0.7888 \\
HLA DSA positive $(n=44)$ & $2.80 \pm 2.44$ & 0.2936 & $39.81 \pm 28.33$ & 0.4273 & $12.61 \pm 7.07$ & 0.5980 \\
Class I DSA positive $(n=19)$ & $2.56 \pm 2.96$ & 0.6867 & $47.49 \pm 32.30$ & 0.8013 & $12.66 \pm 8.43$ & 0.7215 \\
Class II DSA positive $(n=32)$ & $2.85 \pm 1.95$ & 0.1925 & $36.04 \pm 26.74$ & 0.2097 & $12.62 \pm 6.04$ & 0.6220 \\
MICA Abs positive $(n=31)$ & $2.40 \pm 1.43$ & 0.8347 & $40.43 \pm 26.19$ & 0.5140 & $11.97 \pm 5.42$ & 0.4008 \\
\hline
\end{tabular}

${ }^{*} P$ indicates the difference with antibody -negative patients

\section{Risk factors for anti-HLA DSA production assessed by Cox proportional hazard regres- sion analyses}

The Cox proportional hazard method was used to examine the relationship between the presences of anti-HLA DSA among different subpopulations of patients (Table 5). Univariate analysis of risk factors for development of anti-HLA DSA showed patients' age at transplant and eGFR at the time of testing to be insignificant $(P>0.1)$. Two variables of recipient gender and immunosuppression were also not significantly associated with the development of anti-HLA DSA on univariate analysis if $P<0.05$ was considered as significant, but the type of transplantation and HLA mismatch number were significantly associated with the production of anti-HLA DSA $(P<0.05)$. Four covariables with $P<0.1$ on univariate analysis were introduced into a backward stepwise Cox regression model (multivariate analyses) and the results suggested that the type of transplantation and HLA mismatch number were significant negative risk factors for the development of anti-HLA DSA $(P<0.05)$.

\section{DISCUSSION}

The Luminex platform was used in this study to detect HLA and MICA Abs of post-transplant serum samples in a group of long-term survival patients with renal allo-graft. The prevalence of anti-HLA and MICA Abs as well as the relationship between humoral responses and allo-graft function were also analyzed in order to understand the meaning of anti-HLA/MICA antibody moni- toring and to predict the risk of graft failure.

The occurrence of HLA Abs, especially HLA DSA, is known to play an important role in renal graft dysfunction and loss by initiating the process of damage and repair in the endothelium of blood vessels. Many earlier studies have also provided numerous evidence showing the importance of anti-HLA antibodies to allo-graft longevity. However, we should interpret the antibody monitoring results with caution, especially for patients

Table 5 Cox proportional hazard analysis of risk factors for HLA DSA development

\begin{tabular}{lccc}
\hline Variable & Hazard ratio & $P$ & $95 \% \mathrm{CI}$ \\
\hline Univariate analysis & & & \\
Female gender & 0.616 & 0.058 & $0.373-1.017$ \\
\hline Age at transplant & 0.990 & 0.435 & $0.965-1.105$ \\
\hline Living donor & 2.189 & 0.025 & $0.044-0.809$ \\
\hline HLA-ABDRDQ mismatch number & & & \\
$\quad 0-2$ & 1 & & \\
$3-4$ & 1.848 & 0.048 & $1.005-3.398$ \\
\hline $5-6$ & 1.966 & 0.102 & $0.875-4.420$ \\
\hline FK506/MMF/predisone regimens & 2.163 & 0.056 & $0.313-4.014$ \\
\hline eGFR at the time of testing & & & \\
$\quad<30 \mathrm{~mL} / \mathrm{min} / 1.73 \mathrm{~m}^{2}$ & 1 & & \\
\hline $30-60 \mathrm{~mL} / \mathrm{min} / 1.73 \mathrm{~m}^{2}$ & 1.303 & 0.509 & $0.595-2.853$ \\
\hline$>60 \mathrm{~mL} / \mathrm{min} / 1.73 \mathrm{~m}^{2}$ & 1.248 & 0.429 & $0.721-2.158$ \\
\hline Multivariate analysis & & & \\
\hline Female gender & 1.559 & 0.199 & $0.791-3.070$ \\
\hline Living donor & 2.072 & 0.034 & $0.721-3.814$ \\
\hline HLA-ABDRDQ mismatch number & & & \\
\hline $0-2$ & 1 & & \\
\hline $3-4$ & 2.572 & 0.045 & $1.023-6.466$ \\
\hline $5-6$ & 4.409 & 0.003 & $1.631-11.924$ \\
\hline FK506/MMF/predisone regimens & 2.060 & 0.247 & $0.607-6.992$ \\
\hline
\end{tabular}


with normal-function grafts as suggested by Bartel et al. ${ }^{[17]}$. In this study, all 110 enrolled patients received renal transplants for at least 5 years. AntiHLA antibody-positive patients, even DSApositive patients, had comparable Scr, eGFR and BUN levels with antibody-negative patients (Table 4). Between patients with and without normal graft function, there was no statistically significant difference no matter what their antibody statuses were. Thus, it's clear that allo-grafts can survive for many years in the presence of antibodies. Although many groups had demonstrated the presence of DSA is associated with a significantly decreased graft survival, there were some other papers which showed a similar incidence of rejection, similar serum creatinine levels and a similar graft survival in patients with and without DSA ${ }^{[18]}$. Possible reasons for this include: the border between positive and negative reactions differs between centers and amongst studies; some DSA is non-complement fixed, which doesn't mediate rejection; HLA antibodies are frequently observed in the sera from healthy males with no history of allo-sensitization.

This study performed a retrospective analysis of post-transplant anti-HLA and/or MICA Abs development in a cohort of 110 long-term survival renal transplantation patients. Among them, 44 $(40 \%)$ patients developed anti-HLA DSA Abs after transplantation. According to an investigation of former studies, the prevalence of alloantibodies against donor HLA molecules was highly variable, from $1.6 \%$ to $60 \%$ of the patients ${ }^{[19]}$. This great variation is most likely due in part to a different MFI cut-off value, which is used to distinguish the positive from negative result and which is different, being set by various studies and authors ${ }^{[20]}$. Many centers use a threshold of over 1,000 for HLA DSA positivity which results in a close apparent association between the development of DSA and graft damage. However, a few studies with a lower MFI threshold of $\geqslant 300^{[21]}$ or even $\geqslant 100^{[22]}$, for DSA positivity also demonstrated the clinical importance of anti-HLA DSA Abs to graft function and failure. Unfortunately, there is no a consensus at the moment on MFI cut-off value, even though it is highly desirable for solid organ transplantation to have standardized guidelines for testing and management of DSA ${ }^{[23]}$. The higher frequency of antiHLA DSA Abs (40\%) in this study may have the following two reasons. Firstly, the positive reaction was set as NBG ratios $>2.2$ rather than a certain MFI value. Secondly, kidney transplantations of all patients involved in this analysis were carried out at least 5 years ago and the chance of developing anti-HLA DSA Abs would be increased over time as suggested by Lee et al. ${ }^{[24]}$.

Although the number of patients with differing antibody statuses in the graft functioning group had no statistically significant difference with those in the graft dysfunction group, the average MFI was higher in the latter group for those patients with anti-HLA DSA, Class I, DSA, Class II DSA and MICA antibodies. Importantly, the difference between the two groups had a statistical significance in terms of anti-HLA DSA Abs $(P=0.0086)$. The lack of statistical significance for Class I, DSA, Class II DSA and MICA may have resulted from small sample volume and large standard deviation. These results repeatedly indicate the big challenge in determining the cut-off value of MFI for HLA DSA positivity.

Alternatively, previous studies have suggested that antibodies against MICA also have an adverse effect on graft survival, as MICA Abs could be detected among $37 \%$ patients without anti-HLA antibodies and whose grafts had failed ${ }^{[9]}$. However, in this study, we didn't find a correlation between MICA anti-Abs and allo-graft function.

The occurrence of post-transplant NDSA also had adverse effects on renal allo-grafts as shown in an earlier study ${ }^{[25]}$, but there was no evidence showing a significant relationship of NDSA with renal allo-graft function in this analysis, because the average MFI of NDSA in the graft functioning group was comparable with that of NDSA in the graft dysfunction group.

In summary, our study retrospectively analyzed a group of long-term survival patients with renal allo-transplantation and the results showed that a relatively high proportion of patients produced post-transplant anti-HLA and/or MICA antibodies, some of them with anti-HLA DSA Abs. We did not find a strong association between the occurrence rates of either antibody with renal function, which is in contrast with most previous studies. We believe it's very important to monitor the development of anti-HLA and/or MICA antibodies in patients with renal allo-grafts, so as to offer the timely adjustment of immunosuppression regimens of post-transplantation. Over the last decade, advances in antibody detection, especially after introduction of Luminex single-antigen microsphere technology, have revolutionized our approach to kidney transplantation. However, its high sensitivity, which although is able to detect antibodies earlier, is a double-edge sword for doctors and 
patients. We have to carefully interpret the results and estimate its effect on graft function and survival. Furthermore, the cost of regularly detecting anti-HLA/MCIA antibodies by LABScreen method is relative high for most Chinese patients. So currently, we need much more evidence to assess the importance of anti-HLA/MICA antibody monitoring in the future.

\section{References}

[1] Banasik M, Klinger M. Chronic allograft nephropathyImmunologic and non-immunologic factors. Ann Transplant, 2006, 11:7-10.

[2] Hourmant M, Cesbron-Gautier A, Terasaki PI, et al. Frequency and clinical implications of development of donor-specific and non-donor specific HLA antibodies after kidney transplantation. J Am Soc Nephrol, 2005, 16:2804-12.

[3] Cai J, Terasaki PI. Humoral theory of transplantation: Mechanism, prevention, and treatment. Hum Immunol, 2005, 66(4): 334-42.

[4] Terasaki PI, Ozawa M. Predicting kidney graft failure by HLA antibodies: a prospective trial. Am J Transplant, 2004, 4: 438-43.

[5] Lachmann N, Terasaki PI, Budde K, et al. Anti-human leukocyte antigen and donor-specific antibodies detected by Lumin expost-transplant serve as biomarkers for chronic rejection of renal allografts. Transplantation, 2009, 87: 1505-13.

[6] Lefaucheur C, Loupy A, Hill GS, et al. Pre-existing donor-specific HLA antibodies predict outcome in kidney transplantation. J Am Soc Nephrol, 2010,21:1398-406.

[7] Nankivell BJ, Alexander SI. Rejection of the kidney allograft. N Engl J Med, 2010,363:1451-62.

[8] Loupy A, Hill GS, Jordan SC. The impact of donorspecific anti-HLA antibodies on late kidney allograft failure. Nat Rev Nephrol, 2012, 8: 348-57.

[9] Terasaki PI, Ozawa M, Castro R. Four-year follow-up of a prospective trial of HLA and MICA antibodies on kidney graft survival. AmJ Transplant, 2007, 7: 408-15.

[10] Zou Y, Stastny P, Susal C, et al. Antibodies against MICA antigens and kidney-transplant rejection. $N$ Engl J Med, 2007, 357:1293-300.

[11] Zwirner NW, Fernandez-Vina MA, Stastny P. MICA, a new polymorphic HLA related antigen, is expressed mainly by keratinocytes, endothelial cells, and monocytes. Immunogenetics, 1998, 47:139-48.

[12] Zwirner NW, Dole K, Stastny P. Differential surface expression of MICA by endothelial cells, fibroblasts, keratinocytes, and monocytes. Hum Immunol, 1999, 60:323-30.

[13] Molinero LL, Domaica CI, Fuertes MB, et al. Intracellular expression of MICA in activated CD4 T lymphocytes and protection from NK cell-mediated MICA-dependent cytotoxicity. Hum Immunol, 2006, 67:170-82.

[14] Terasaki PI. Humoral theory of transplantation. Am J Transplant, 2003, 3:665-73.

[15] Terasaki PI, Cai J. Humoral theory of transplantation: furtherevidence. Curr Opin Immunol, 2005, 17: 541-5.

[16] Schwartz GJ, Gauthier B. A simple estimate of glomerular filtration ratein adolescent boys. J Pediatr,1985, 106:522-6.

[17] Bartel G, Regele H, Wahrmann M, et al. Post-transplant HLA allo-reactivityin stable kidney transplant recipients-incidences and impact on long-termallograft outcomes. Am J Transplant, 2008, 8:2652-60.

[18] Aubert V, Venetz JP, Pantaleo G, et al. Are alldonorspecific antibodies detected by solid-phase assaybefore transplantation clinically relevant? Transplantation,2009, 87: 1897-902.

[19] Akalin E, Pascual M. Sensitization after kidney transplantation. Clin J Am Soc Nephrol, 2006, 1: 433-40.

[20] Roelen DL, Doxiadis II, Claas FH. Detection and clinical relevance of donor specific HLA antibodies: a matter of debate. TransplInt, 2012, 25: 604-10.

[21] Wiebe C, Gibson IW, Blydt-Hansen TD, et al. Evolutionand clinical pathologic correlations of de novo donor-specificHLA antibody post kidney transplant. Am J Transplant,2012, 12: 1157-67.

[22] Colvin RB. Antibody-mediated renal allograft rejection: diagnosis and pathogenesis. J Am SocNephrol, 2007, 18:1046-56.

[23] Tait BD, Susal C, Gebel HM, et al. Consensus guidelines on the testing and clinical management issues associated with HLA and non-HLA antibodies in transplantation. Transplantation, 2013, 95: 19-47.

[24] Lee PC, Zhu L, Terasak PI, et al. HLA-specific antibodies developed in the first year post-transplant are predictive of chronic rejection and renal graft loss. Transplantation, 2009, 88: 568-74.

[25] Lachmann N, Terasaki PI, Schönemann C. Donor-specific HLA antibodies in chronic renal allograft rejection: A prospective trial with a four-year follow-up. Clin Transpl, 2006, 2006:171-99.

(Received 31 December 2017, Revised 19 January 2018, Accepted 24 January 2018) 
52 The incidence and clinical relevance of anti-HLA and/or MICA antibodies in patients with long-term survival renal allo-grafts, 2018, 2(1) 